\title{
Habilidades comunicacionales en la entrevista clínica
}

\author{
Communication abilities in the clinical interview
}

\author{
A. Martínez Rodríguez, R. Fernández Cervantes, F. Medina i Mirapeix, I. Raposo \\ Vidal, S. Viñas Diz, M. Chouza Insua
}

\begin{abstract}
Resumen
Las habilidades comunicacionales constituyen una parte importante de la entrevista clínica. Para una mejor comprensión pueden dividirse en los siguientes apartados: contexto, escucha, comprensión, estrategia y resumen general. Se detallan en cada uno de ellos (a excepción del contexto por haber sido abordado en otro artículo) una serie de técnicas o consideraciones de interés que facilitan una mejora en la interacción del profesional de la salud (fisioterapeuta) con el consultante. Esta mejora repercute tanto en una mayor calidad de los resultados como a nivel afectivo y emocional en ambos protagonistas de la relación.
\end{abstract}

\begin{abstract}
The communication skills are an important part of the clinical interview. To get a better comprehension they can be divided into the following steps: context, listening, acknowledgement, strategy and summary. Some techniques or interesting considerations which can improve health care provider (physiotherapist) patient relationship are explained in each one (but the context because it has been considered in another article). This improvement is involved with not only better clinical outcomes, but also affective and emotional level for both relationship's main characters.
\end{abstract}

\section{Palabras clave}

Fisioterapeuta; Habilidades comunicacionales; Relación; Paciente.

Keywords

Physiotherapist; Communication skills; Relationship; Patient.

\section{INTRODUCCIÓN}

Un avance importante en la concepción de la salud la estableció la Organización Mundial de la Salud al reconocer como integrantes de la misma las facetas psicológica y social con el fin de realizar un abordaje integral de la persona ${ }^{1,2}$. Sin embargo a pesar del re- conocimiento de que ha gozado esta perspectiva, ha quedado fundamentalmente relegada a un plano teórico sin gran trascendencia en el abordaje clínico diario, que ha seguido mayormente el modelo biomédico tradicional ${ }^{3-9}$.

En la actualidad el énfasis en la calidad ha puesto de manifiesto que además del aspecto científicotécnico es preciso atender al aspecto relacional ya que también es determinante para el logro de una mayor efectividad ${ }^{10-15}$ y eficiencia ${ }^{16}$, tanto a nivel terapéutico como preventivo ${ }^{17}$. Aspectos tan variados como la satisfacción, el cumplimiento, la percepción de la competencia profesional e incluso el pronóstico de la enfermedad o el estado de salud general están relacionados con las habilidades de comunicación del profesional sanitario ${ }^{15,18-21}$.

En Fisioterapia, la relación asistencial tiene particular relevancia, ya que los fisioterapeutas a menudo se encuentran muy cerca de sus pacientes, tanto física como mentalmente ${ }^{22}$. Sin embargo, los fisioterapeutas perciben deficiencias a la hora de establecer una buena relación con los pacientes ${ }^{22}$. Por otra parte se ha evidenciado que las carencias de comunicación que constituyen un $34 \%$ del total de los problemas señalados por los usuarios de Fisioterapia para el incumplimiento de la terapia ${ }^{18}$. Parece que los fisioterapeutas son empáticos, pero que no favorecen la participación activa. Con relación a ésta se sabe, por ejemplo, que los pacientes aceptan, recuerdan y siguen más las instrucciones y consejos cuando sus expectativas son consideradas ${ }^{18}$, sin embargo, los fisioterapeutas las contemplan sólo en el $12 \%$ de las sesiones y sólo se interesan por los tratamientos aplicados o actuales en el $4 \%$ de los casos. 
A pesar de la importancia de la relación interpersonal en la calidad de la atención y de las evidentes carencias que se reconoce que existen en los fisioterapeutas, según ellos mismos ${ }^{22}$ y los pacientes ${ }^{18}$, el mayor acento de la formación de pregrado y continuada de estos profesionales se ha enfatizado en la vertiente científico-técnica, dejando de lado la formación en los aspectos comunicacionales y emocionales $^{23}$.

Probablemente a esta priorización haya contribuido la inexistencia de textos específicos sobre técnicas comunicacionales en Fisioterapia ${ }^{24}$. Este artículo tiene el objetivo de presentar una revisión realizada sobre técnicas que pueden ser útiles en la entrevista clínica, con especial énfasis en los factores que se deben considerar para las principales tareas a realizar en una entrevista clínica.

\section{Material y método}

La información obtenida tras una revisión bibliográfica se presenta en tres apartados principales. El primero versa sobre los modelos de orientación de la entrevista clínica, el segundo sobre las fases y tareas de que se compone la entrevista y por último se explican y desarrollan los factores a considerar para garantizar su buen desarrollo.

\section{Resultados}

\section{Modelos de orientación de la entrevista}

Con relación a las estrategias globales de abordar la entrevista existen dos modelos fundamentales de acuerdo al sujeto sobre el que se orienta: a) la orienta- da al paciente (se asume que el conocimiento y la experiencia del paciente son la base para activar sus recursos), y b) la orientada al fisioterapeuta (es el cono- cimiento y experiencia del fisioterapeuta el que activa los recursos propios del paciente) ${ }^{24}$. Similares conceptos se encierran en los modelos de relación que Ozar distingue (además del de empresa comercial): «modelo gremial»y «modelo interactivo» ${ }^{25}$.

El modelo orientado hacia el clínico está basado en la postura que consideraba al buen profesional de salud como al paternalista y autoritario que obraba según el principio de beneficencia (dando lo mejor para el paciente pero sin contar con él) ${ }^{19}$

La entrevista centrada en el consultante tiene en cuenta, junto a los problemas del usuario, sus ideas, expectativas $^{6,9,26,27}$ y sentimientos ${ }^{14}$, Se considera a las emociones como integrantes y responsables de problemas de salud y se informa al paciente para que sea capaz de autorresponsabilizarse de su propia salud ${ }^{19}$ («Dame un pez y comeré hoy. Enséñame a pescar y comeré durante toda la vida») ${ }^{25}$ en la medida en que ésta depende de él, aunque no se excluyen posiciones más paternalistas o autoritarias de ser requeridas por el usuario, que de esta forma adopta un papel más pasivo ${ }^{2,14,26}$. Esta flexibilidad no anula la búsqueda de alternativas para lograr su implicación cuando ésta conlleve una posible mejora en el estado de salud ${ }^{28}$. Las personas con menor tendencia participativa son ancianos, pacientes con procesos de cierta gravedad, menor nivel cultural, etc. ${ }^{29}$.

Actualmente se está pasando de la orientación paternalista a la consideración de la libertad como elemento central, estableciéndose la toma decisiones conjunta o compartida como ideal a conseguir ${ }^{14} 19$,

${ }^{29}$. Es en este marco donde la entrevista orientada al paciente adquiere su mayor relevancia.

\section{Fases y tareas de una entrevista}

La entrevista clínica puede abordarse desde un punto de vista estructural y desde una perspectiva funcional. A nivel estructural observamos dos estadios funda- mentales: fase exploratoria y fase resolutiva $2,9,10,20,30$ con los siguientes componentes:

\section{Fase exploratoria}

Tratamos de comprender cuál es el problema del consultante y se utilizan técnicas que permiten el vaciado de información por su parte:

- Establecimiento de una relación en un clima de confianza.

- Profundización en el motivo de consulta; diagnóstico fisioterapéutico:

- Anamnesis.

- Exploración. 


\section{Fase resolutiva}

Se intenta llegar a un acuerdo para la solución del problema:

- Información

- Negociación

- Resumen y comprobación

Desde un punto de vista funcional podemos distinguir una serie de tareas que están interconectadas y son difíciles de separar ${ }^{30,31}$. En la tabla 1 se especifican las principales tareas.

Tabla 1. Principales tareas de una entrevista

\begin{tabular}{|c|c|}
\hline Enganchar & Establecer un buen contacto con el cliente \\
\hline Empatizar & Expresar solidaridad emocional \\
\hline Educar & $\begin{array}{l}\text { Abordar actitudes, conocimientos y conductas para adecuadas a un fin } \\
\text { terapéutico o preventivo. Evaluar la comprensión y permitir ser preguntado }\end{array}$ \\
\hline Expectativas & $\begin{array}{l}\text { Explorar y tener en cuenta las creencias y opiniones del paciente acerca de su } \\
\text { estado y lo que procede hacer. Se ha de demostrar que ha entendido las } \\
\text { expectativas del paciente }\end{array}$ \\
\hline Enrolar & Implicar al paciente en su proceso asistencial \\
\hline
\end{tabular}

Factores a considerar en las tareas de una entrevista

Los factores que consideran las tareas funcionales descritas y que contribuyen a un correcto desarrollo de la entrevista son las siguientes ${ }^{30}$ :

- Contexto o entorno que favorezca la entrevista. Se tendrá en cuenta la imagen del profesional, la proxémica, el lenguaje del cuerpo y los componentes paralingüísticos.

- Escucha. El fin es el de favorecer la narración del paciente.

- Comprensión. Captar y reconocer lo que el paciente siente y manifiesta.

- Estrategia. Elaborar un plan de acción conjuntamente con el paciente.

- Resumen general. Se hace un breve esquema de lo visto y lo que se va a realizar en un futuro.

A continuación se expone con mayor detalle los contenidos de cada uno de ellos junto con las técnicas que se han identificado como más relevantes para poderlos llevar a cabo. No se expone el contexto por haber sido abordado en un artículo previo ${ }^{32}$.

\section{Escucha}

En primer lugar se debe establecer la duración de la entrevista ${ }^{31}$ con el fin de favorecer que el consultante no empiece a hablar de asuntos importantes cuando ha finalizado el tiempo de que disponemos para él. Se evita de esta forma que el fisioterapeuta se ponga nervioso por tener otras cosas que hacer y que el paciente piense que no existe interés por sus problemas o que no merecen la pena ${ }^{13,33}$. Saber escuchar no es sólo oír un listado de cosas, se trata de adoptar una posición abierta ${ }^{21,34}$ y activa y permitir que el paciente pueda exponer el problema sin interrumpirle precozmente, ni realizar conclusiones prematuras $^{10,35}$, es ser empáticos ${ }^{7}$ y facilitarle que exprese cuáles son sus sentimientos, sus expectativas, sus esperanzas y cómo afecta la enfermedad a su vida ${ }^{20}$.

Algunas de las técnicas son:

Formular preguntas abiertas ${ }^{11,13,15,20,30}$

Por ejemplo: «¿Me podría decir cómo es el dolor que siente?». Si no funciona, se debe intentar con un menú de sugerencias que incluya al menos dos opciones ("¿es un dolor continuo o es más repentino y punzante?») y como último recurso se pueden usar las preguntas cerradas ("¿Le duele de forma persistente, continuada?»), aunque estas últimas pueden ser de primera elección en caso de buscar información muy concreta ${ }^{10}$. 
Cuanto menos esfuerzo requiere el paciente para responder a una pregunta tanto menos fiable resulta la respuesta. Al paciente le interesa más quedar bien que proporcionar información sobre su problema de salud. Además las preguntas cerradas no permiten expresar matices y favorecen el interrogatorio inducido (las respuestas que estaba buscando el terapeuta) ${ }^{10,36}$, ya que lo que puede decir el consultante está fuertemente influenciado por el tipo de preguntas que hace el fisioterapeuta y cómo las hace ${ }^{37}$

\section{Facilitar}

Conducta verbal y no verbal que ayuda a iniciar o proseguir el relato sin sugerir contenidos ${ }^{34}$.

- Silencio. Ausencia de comunicación verbal que tiene por efecto proporcionar un tiempo de meditación al entrevistado ${ }^{35}$, ayudar a su concentración o actuar como catalizador de determinadas reacciones emocionales en el curso de la entrevista ${ }^{10}$. Se debe dejar un cierto tiempo de latencia entre la finalización de la frase del paciente y la intervención del fisioterapeuta, contrariamente a lo que hacen profesionales de alta reactividad que pretenden controlar de este modo la relación ${ }^{14}$, El fisioterapeuta atento obtiene información de esta manera, que le permite adaptarse a la situación o modificar la orientación inicial ${ }^{13}$.

- Frases y gestos sencillos: uhm, mmm, cuéntame más ...

- Repetición de palabras clave: repetición de una palabra o frase que orienta la atención del paciente hacia un determinado contenido. Por ejemplo: el paciente dice «el dolor me llegaba hasta la punta de los dedos»; el fisioterapeuta podría decir: «¿la punta de los dedos?» Deben formularse en una tonalidad adecuada (por ejemplo, no con incredulidad), con empatía y respeto. No abusar de ellas porque pueden provocar el cansancio o irritación del entrevistado ${ }^{10}$.

\section{Clarificar}

Aclarar y explicitar lo dicho por el paciente ${ }^{15,30}$. Conducta verbal: ¿qué quiere decir con ... ?, o conducta no verbal (expresión de extrañeza) que obliga a explicar el sentido de un término o idea. Igualmente emplear en el tono adecuado y no abusar de ellas como si se tratase de un interrogatorio policíaco $^{10}$.

\section{Señalamientos}

Poner de manifiesto o mostrar al paciente emociones o conductas, profundizando en las motivaciones del paciente y evidenciando conflictos o áreas de estrés psicosocial. Ayuda al consultante a clarificar emociones/ideas. Por ejemplo sería decir: «parece como si se sintiera .... (observación del estado de ánimo), o «desde hace una semana observo que ... » (relativo a conductas) ${ }^{10}$.

\section{Compresión}

Captar y reconocer lo que el paciente siente y manifiesta. La respuesta empática consta de tres pasos: identificar la emoción, la causa y transmitir su comprensión ${ }^{10,15}$. Si no sabemos qué siente el paciente hay que realizar preguntas abiertas hasta identificar el sentimiento Con la respuesta empática se puede:

- Explorar la emoción del paciente,

- Validar o legitimar sus sentimientos ${ }^{17}$. Es recomendable una vez que se ha comprendido «normalizar», sabiendo que es factible aún sin estar de acuerdo con el punto de vista del paciente $^{15,30}$,

- Además también se puede utilizar la validación de sentimientos cuando nosotros estamos experimentando un sentimiento intenso. Hay que describir en vez de mostrado y explicando en vez de exhibirlo ${ }^{30}$.

\section{Estrategia}

Elaborar un plan de acción conjuntamente con el paciente. Hay que tener en cuenta ${ }^{30}$ :

- Plan fisioterapéutico ideal

- Expectativas del paciente. 
Es posible averiguar creencias y conocimientos del paciente, así como las expectativas que pudiera tener sobre el régimen preventivo o terapéutico. Este proceso se denomina bidireccionalidad de la información ${ }^{2}$.

Antes de detallar las técnicas, decir que una premisa fundamental en este proceso será la de utilizar en la medida de lo posible, vocabulario neutro (de bajo contenido emocional como, por ejemplo, decir persistente en vez de crónico) ${ }^{10}$. Además evitar términos excesivamente cultos o científicos, siempre adaptados al consulrante ${ }^{13,19,21,35}$.

\section{Enunciación}

Anunciar los contenidos que se explicarán. Prepara al paciente para recibir los contenidos que le van a ser transmitidos. Ejemplo: en primer lugar le informaré sobre las características de su enfermedad y a continuación del tratamiento. También sirve para poder hablar un rato sin interrupciones ${ }^{10}$.

\section{Ejemplificación y complemento visual-táctil}

Para informar de la conducta terapéutica o preventiva. Explicar un concepto mediante un ejemplo de fácil comprensión. Aproxima al paciente a los mecanismos por los que actúa la enfermedad o el tratamiento.

Debe ser clara, breve, concisa y de escasa complejidad, sobre todo cuando va dirigida a niños, ancianos con cierto déficit intelectual o personas de escasa instrucción/formación ${ }^{10}$.

\section{Racionalidad}

Explicar el fundamento de la conducta terapéutica o diagnóstica propuesta ${ }^{10}$.

\section{Detallar/escribir cambios}

Detallar las instrucciones y cambios conductuales propuestos, si hace falta por escrito ${ }^{10}$.

\section{Comprobar asimilación}

Cerciorarse de la exacta comprensión de los mensajes. Ejemplo: ¿lo ha entendido todo? ¿puede repetir- me ... ? $?^{2,10,15,30,35}$.

\section{Propuesta de acuerdo}

Si la persona se siente partícipe de las medidas terapéuticas y/o preventivas será más probable que las lleve a cabo. Por ello es importante como intervención mínima asegurarse que está de acuerdo con las medidas propuestas. El desacuerdo se puede percibir por microexpresiones de desagrado o evitación o con frases del tipo «no sé, no sé». En tal caso la primera respuesta del fisioterapeuta será evaluativa (indagar sobre su opinión) y no justificativa ${ }^{2}$, utilizando un señalamiento («no parece muy convencido») o explorando creencias («¿cuál es su opinión al respecto?») y reconversión de ideas (aclarando conceptos, etiologías de los diversos procesos que afectan la salud, etc. $)^{10}$.

Se debe tener en cuenta a la familia y/o soporte social del paciente ${ }^{2,10,38,39}$, así como los recursos con los que cuenta con el fin de llegar a una solución realista y lo más activa posible del problema.

Otras técnicas que involucran al consultante $\operatorname{son}^{10}$ :

- La cesión (intencional «le agradezco su franqueza y lo tendré en cuenta para otras veces» o real «en efecto, podemos considerarlo»).

- Doble pacto «yo tendré en cuenta esa posibilidad, pero le propongo que entretanto realice estos ejercicios».

También se puede realizar un paréntesis «vamos a dejado por el momento», cuando el profesional considere importante no abordar la posibilidad sugerida al menos inicialmente.

Las instrucciones por escrito son recomendables, sobre todo en casos en que el consultante pueda tener problemas para recordar lo pactado ${ }^{10}$.

\section{Resumen general}

Se hace un breve esquema de lo visto y lo que se va a realizar en un futuro ${ }^{10,30}$. Ayuda a disminuir los malos entendidos y a clarificar conceptos ${ }^{13,34,35}$. 


\section{Conclusiones}

Las habilidades comunicacionales imprescindibles en el aspecto relacional son numerosas y aunque algunas puedan parecer obvias o sencillas su complicación no reside tanto en su ejecución simple como en su integración en la rutina diaria y en el cambio del rol del fisioterapeuta y del paciente o cliente que implican.

Dichos aspectos facilitan la obtención e intercambio de información, además de mejorar la interacción con el usuario, dotando al fisioterapeuta de flexibilidad y capacidad de adaptación a las circunstancias específicas del paciente, del contexto y de su propia persona. De este modo es posible disminuir o minimizar el estrés al que está sometido por el desempeño de su labor.

El conocimiento de las habilidades comunicacionales no garantiza su utilización, pero constituye un primer paso para su aplicación tanto a nivel terapéutico como preventivo.

\section{Bibliografía}

1. Frías Osuna A. Salud pública y educación para la salud. Barcelona: Masson; 2000.

2. Martín Zurro A, Cano Pérez JE Atención Primaria. Conceptos, organización y práctica clínica. Barcelona: Doyma Libros; 1994

3. Pera Blanco Morales . El concepto de salud y nuevas patologías. En: La formación de los profesionales de la salud. Escenarios y factores determinantes. Madrid: Fundación BBV; 1998.

4. Alfonso Roca MT, Álvarez-Dardet Díaz. Enfermería. Enfermería comunitaria I. Serie manuales de enfermería. Barcelona: Ediciones Científicas y Técnicas. Masson-Salvat Enfermería.

5. Weiss SC. Humanities in medical education: revising me doctor-patient relationship. Med Law 2000; 19(3):55967.

6. Greco M, Francis W, Buckley J, Brownlea A, McGovern J. Real-patient evaluation of communication skills teaching for GP registrars. Family practice 1998;15(1):51-7.

7. McLeod ME. Doctor-patient relationship: perspectives, needs, and communication. The American Journal of Gastroenterology 1998;93(5):676-80.

8. Abramovitch H, Schwartz E. Three stages of medical dialogue. Theoretical Medicine 1996;17(2):175-87.

9. Torío Durántez J, García Tirado Me. Valoración de la orientación al paciente en las consultas médicas de Atención Primaria. Atención Primaria 1997;20(1):17-23.

10. Borrell i Carrió F. Manual de entrevista clínica. Barcelona: Doyma; 1989.

11. Borrell i Carrió F Tareas a cumplimentar en la entrevista clínica. Revista de Medicina Familiar y Comunitaria 1991; 1 (1):32-6.

12. Quesada Jiménez F, Martínez-Cañavete T. Formación en habilidades de comunicación a médicos residentes de medicina familiar y comunitaria. Editoriales. Atención Primaria 1991;19(5):219-20.

13. Gallego Izquierdo T, Yuste Sánchez MJ. La entrevista en Fisioterapia. Docencia. Fisioterapia 1996;18(3):172-6.

14. Ong LML, de Haes JCJM, Hoos AM, Lames FB. Doctor-patient communication: a review of the literature, Social Science and Medicine 1995;40(7):903-18

15. Simpson M, Buckman R, Stewart M, Maguire P, Lipkin M, Novack D, Till J. Comunicación médico-paciente: el informe del consenso de Toronto. BMJ 1993;8(1):40-5.

16. Sturmberg JP. Continuity of care: towards a definition based on experiences of practising GPs. Family Practice 2000; 17(1): 16-20.

17. Dubé CE, O'Donell JF, Novack DH. Communication skills for preventive interventions. Academic Medicine 2000;75(7 Suppl):S45-54.

18. Escolar Reina P, Medina i Mirapeix F, González Pérez. La dimensión educativa de la Fisioterapia: una oportunidad para mejorar la calidad asistencial. Fisioterapia 1997;19 monográfico:63-74.

19. Torío Durántez J, García Tirado Me. Relación médico-paciente y entrevista clínica (y II): opinión y preferencias de los médicos. Atención Primaria 1997;19(1):27-34.

20. Castro Gómez JA, Quesada Jiménez F. Cómo mejorar nuestras entrevistas clínicas. Atención Primaria 1996;18(7):399-402.

21. Virshup BB, Oppenberg AA, Coleman MM. Strategic Risk Management: Reducing Malpractice Claims Through More Effective Patient-Doctor Communication. American Journal of Medical Quality 1999;14(4):153-9.

22. Dahlgren MA, Almsquit A, Krook J. Physiotherapists in Balint group training. Physiotherapy Research International 2000;5(2):85-95.

23. Maxwell M, Dickson D. An evaluation of communication skills training for physiotherapy students, Medical Teacher 1991; 13(4):333-8).

24. Westman Kumlin I, Kroksmark T. The first Encounter, Physiotherapists Conceptions of Establishing Therapeutic Relationships. Scand J Caring Sci 1992;6(1):37-44.

25. Bellner AL. Senses of responsibility. A Challenge for Occupational and Physical Therapists in the Context of Ongoing Professionalization. Scand J Caring Sci 1999; 13( 1):55-62.

26. Torío Durántez J, García Tirado MC. Relación médico-paciente y entrevista clínica (I): opinión y preferencias de los usuarios. Atención Primaria 1997;19(1):18-26.

27. Britten N, Stevenson FA, Barry CA, Barber N, Bradley CP. Misunderstandings in prescribing decisions in general practice: qualitative study, BMJ 2000;320(7233):484-8. 
28. Kaplan SH, Gandek B, Greenfield S, Rogers W, Ware JE. Patient and Visit Characteristics Related to Physician' s Participatory Decision-Making Style. Medical Care 1995;33(12): 1176-87.

29. Benbassat J, Pilpel D, Tidhar M. Patients preferences for participation in clinical decision making: a review of published surveys. Behavioral Medicine 1998;24(2):81-8.

30. Buckman R, Korsch B, Baile W, 2000. Guía Práctica de Habilidades de comunicación en la práctica clínica. Toronto: Medical Audiovisual Communications Inc/Fundación de Ciencias de la Salud; 2000.

31. 31. Nisselle P. Difficult doctor-patient relationships. Australian Family Physician 2000;29(1):47-9.

32. Martínez A, Patiño S, Paseiro G, Martínez Sánchez N, Fernández R. Influencia del contexto en la calidad de la entrevista clínica. Revista Iberoamericana de Fisioterapia y Kinesiología 2001;4(2):75-80.

33. Cibanal Juan L. Interrelación del profesional de enfermería con el paciente. Barcelona: Doyma; 1991.

34. Albaiges i Sans Ll. Entrevista clínica. Atención Primaria 1991; 8(1):40-7.

35. Gorney M. The role of communication in the physicians office, Clinics in Plastic Surgery 1999;26(1): 133-4l.

36. Thornquist E. Examination and Communications: a Study of First Encounters Between Patients and Physiotherapists. Family Practice 1992;9(2):195-202.

37. Thornquist E. Varieties of functional assessment in physiotherapy. Scandinavian Journal of Primary Health Care 1994b; 12: 44-50.

38. Inui TS. Establishing the doctor-patient relationship: science, art or competence? Schwiz Med Wochenschr 1998;128(7):225-30.

39. Ryan NP, Wade JC, Nice A, Shenefelt H, Shepard K. Physical therapists perceptions of family involvement in the rehabilitation process. Physiotherapy Research International 1996; 1 (3): 159-79. 